\title{
Research
}

\section{Possible missed opportunities for diagnosing colorectal cancer in Dutch primary care:}

\author{
a multimethods approach
}

\begin{abstract}
Background

Early detection of colorectal cancer (CRC)

is important to achieve better survival.

Discriminating symptoms suggestive of CRC from benign conditions is a challenge for GPs because most known 'alarm symptoms' have low predictive values.
\end{abstract}

\section{Aim}

To further understand the diagnostic process of $\mathrm{CRC}$ in general practice in terms of healthcare use and by analysing factors related to diagnostic intervals.

\section{Design and setting}

A multimethod approach comprising a historical, prospective registry study and qualitative content analysis.

\section{Method}

Healthcare use in the year before referral for colonoscopy was compared between patients diagnosed with CRC and an age-, sex,- and GP-matched control population. Qualitative content analysis was performed on free texts in electronic patient records from a purposive sample of patients with CRC.

\section{Results}

Patients with CRC ( $n=287)$ had $41 \%(25-59 \%)$ more face-to-face contacts and $21 \%$ (7-37\%) more medication prescriptions than controls ( $n=828)$. Forty-six per cent of patients with CRC had two or more contacts for digestive reasons, compared with $12.2 \%$ of controls, more often for symptoms than diagnoses. From qualitative analysis two themes emerged: possible missed diagnostic opportunities and "improvements in diagnostic process unlikely". Possible missed diagnostic opportunities were related to patients waiting before presenting symptoms, doctors attributing symptoms to comorbid conditions or medication use, or doctors sticking to an initial diagnosis.

\section{Conclusion}

Fewer missed diagnostic opportunities might occur if GPs are aware of pitfalls in diagnosing CRC: the assumption that symptoms are caused by comorbid conditions or medication or relating complaints to pre-existing medical conditions. GPs also need to be aware that repeated digestive complaints warrant rethinking an earlier diagnosis.

\section{Keywords}

colorectal tumours; early diagnosis; general

practice; neoplasms; primary health care.

\section{INTRODUCTION}

Worldwide, colorectal cancer (CRC) is the third most commonly diagnosed cancer in males and the second in females, with the highest incidence rates occurring in Western countries. ${ }^{1}$ Although screening programmes have been implemented in many European countries, including the Netherlands and the UK, the majority of new cases will present symptomatically and outside screening programmes. ${ }^{2-4}$ In countries where the GP acts as a gatekeeper referring to specialty care, the $\mathrm{GP}$ is challenged to adequately discriminate between symptoms possibly caused by CRC and symptoms caused by benign conditions. This is important because detection of CRC at earlier tumour stages leads to more treatment with curative intent and has been associated with better survival. ${ }^{5-6}$

The diagnostic interval (the period between first presentation and diagnosis) can be prolonged in patients with CRC.,8 'Alarm' symptoms for CRC include rectal blood loss, constipation, diarrhoea, abdominal pain, abdominal tenderness, and weight loss. ${ }^{9.10}$ However, most of these symptoms are commonly presented in primary care and diagnosing CRC is a relatively rare event for GPs, therefore, these symptoms have low positive predictive values for $\mathrm{CRC} .{ }^{11,12} \mathrm{~A}$ recent international

D Brandenbarg, MSc, PhD candidate;

F Groenhof, MSc, data manager, Registration Network Groningen; IM Siewers, BSc, medical student; A van der Voort, BSc, medical student; AJ Berendsen, MD, PhD, head of research program oncology in primary care, Department of General Practice, University Medical Center Groningen, University of Groningen, Groningen, the Netherlands. FM Walter, MD, FRCGP, principal researcher in primary care cancer research, Primary Care Unit, Department of Public Health and Primary Care, University of Cambridge, Cambridge, UK.

Address for correspondence Daan Brandenbarg, University of Groningen cohort study suggests that all abdominal symptoms presented in general practice require the same degree of attention as classical alarm symptoms, but that further specific information is required due to the unspecific nature of such symptoms. ${ }^{13}$

Recent Scandinavian studies have analysed routinely collected national healthcare data for other factors that might improve the diagnostic process, which showed increased contact rates of patients with CRC in primary care before CRC diagnosis compared with a reference population; reasons for these contacts were not analysed, and neither was prescribed medication. ${ }^{14,15}$ A Danish study also showed increased diagnostic activity in primary care before CRC diagnosis, with patients having more consultations, haemoglobin measurements, and more haemorrhoid prescriptions; other reasons for consultations and medication use were not analysed in this study. ${ }^{16}$

To help GPs to make timely referrals, potentially leading to more timely diagnosis, this study aimed to further investigate the diagnostic process in general practice by performing a multimethods study, combining quantitative and qualitative data from primary care electronic patient records. Specifically, the researchers aimed to compare annual consultation
University Medical Center Groningen, Department of General Practice, Antonius Deusinglaan 1, PO Box 196, 9700 AD Groningen, the Netherlands.

Email: d.brandenbargaumcg.nl

Submitted: 10 August 2017; Editor's response: 15 September 2017; final acceptance: 11 October 2017.

\section{CBritish Journal of General Practice}

This is the full-length article (published online 5 Dec 2017) of an abridged version published in print. Cite this version as: Br J Gen Pract 2017. DOI: https://doi.org/10.3399/bjgp17X693905 


\section{How this fits in}

Discriminating symptoms suggestive of colorectal cancer (CRC) from benign conditions is a challenge for GPs because most known 'alarm symptoms' have low predictive values. This study showed that patients with CRC have more GP contacts and medication prescriptions in the year before referral compared with a matched control population. Qualitative analysis suggested patient factors might lead to missed diagnostic opportunities and doctor factors could contribute to missed diagnostic opportunities.

and medication rates, reasons for these consultations, and type of prescribed medication, between patients developing $\mathrm{CRC}$ and a reference population. In addition, the study aimed to assess the occurrence of, and factors influencing, diagnostic intervals in Dutch general practice, and the presenting symptoms of patients with CRC who consult their GP.

\section{METHOD}

\section{Design}

A multimethods approach, combining quantitative and qualitative data, was used to study healthcare use and the diagnostic process for CRC in Dutch general practice. Quantitative data from a primary care registry concerning healthcare use of patients in the year preceding a CRC diagnosis were compared to an age- and sex-matched reference population from the same GP. A qualitative content analysis was performed of GPs' electronic patient records from a purposive sample of these CRC patients.

Participants and sample. Patients with CRC ( $n=287)$ and matched controls were identified in the Registration Network Groningen (RNG). This network comprises three GP group practices in the northern part of the Netherlands and has a dynamic patient population of approximately 30000 patients. In the Netherlands, all inhabitants are registered with a GP. Apart from a slight over-representation of adults aged 25-44 years and females, the RNG database is comparable to the Dutch population. ${ }^{17}$ CRC patients were selected based on the occurrence of International Classification of Primary Care (ICPC) code D75 (colon or rectum malignancyl in their electronic patient record between 1 January 1999 and 31 December 2014. Diagnoses of CRC were validated using the correspondence with hospital doctors and hospital patient files. Additional information about cancer treatment and stage was also collected. Controls were randomly selected from all patients without a diagnosis of CRC (no ICPC code D75) from the RNG database. Each patient with CRC was individually matched to three controls based on age ( \pm 1 year), sex, and GP, with a minimum of one control per patient. Patients and controls both had to be registered in the GP's practice at the time of $\mathrm{CRC}$ diagnosis. The date of referral by the GP for colonoscopy was used as the index date for matched controls. When this date was not available, the date of the first record of $\mathrm{CRC}$ in the patient record was used.

For qualitative analysis a purposive sample of these patients with CRC was created. The authors sampled purposively on extreme cases in order to obtain as much variation as possible in the sample. Criteria for this sampling procedure were: variation of age, sex, tumour location, tumour stage, and annual number of GP contacts. These criteria were extracted from the RNG database. Without further knowledge of the content of the patients' electronic patient record, initially two patients from each category were selected, one with a high score in that category and one with a low score. This process was iteratively continued until saturation was reached.

Data collection. The RNG GPs follow a standardised protocol for coding of their data. All patient contacts are coded according to ICPC version $1,{ }^{18}$ and medication is coded using the Anatomical and Therapeutical Chemical (ATC) Classification System. ${ }^{19}$ ICPC codes 1-30 are symptom codes, while the higher codes, 70-99, are diagnosis codes. For example, ICPC-D11 is the code for diarrhoea and ICPC-D75 for CRC. In two practices these data are stored using the MicroHis electronic health record system, and one practice uses the Medicom system. All ICPC and ATC codes and the date of coding are stored in the RNG database, as are patients' demographic features. For the purpose of this study all ICPC codes for face-to-face contacts in the year before referral/index date were extracted. Faceto-face contacts consisted of consultations in general practice and visits to patients homes made by GPs or practice nurses. Furthermore, all medication prescriptions coded in the RNG database in the year before referral/index date were extracted. This included all new medications 
prescribed by the GP and also repeated medications for chronic conditions.

GPs also register free texts of their consultations in the electronic patient files according to the SOAP-system. A SOAP journal comprises four data fields: the subjective (S) data field is used to record, in plain text, what the patient describes, such as complaints, symptoms, or reason for encounter; the objective (0) data field is for recording GPs' findings from clinical examination or measurements; the analysis (A) data field is used for coding the diagnosis or most important symptom using the ICPC coding system (GPs mostly use one code per consultation); and the plan (P) data field records GPs' actions in plain text, for example, referrals to medical specialists, prescribed medication (ATC codes), or follow-up appointments. For the purposive sample of patients with CRC, all free text (SOAP) from the year before the referral date were downloaded. These data was anonymised in general practices and files were stored as Portable Document Format (PDF) files for analysis in Atlas/TI (version 7). Figure 1. Process of incl
$C R C=$ colorectal cancer.

\section{Quantitative analysis}

Calculation of contacts and prescriptions, observation time, and rates. As not all patients were registered during the entire study period, the observation time between entry in the RNG database and the date of referral/index date was calculated. For all patients with $\mathrm{CRC}$ and controls the number of face-to-face contacts and medication prescriptions were counted in the year before referral based on the number of registered dates of contact or start dates of medication, respectively.

Median numbers and interquartile ranges (IQR) of contacts and prescriptions were then calculated in the year before referral. Annual rates were calculated by dividing the total number of contacts and prescriptions by the observation time.

ICPC codes associated with face-to-face contacts and ATC codes for prescriptions in the year before diagnosis were extracted from the RNG database. Rates of ICPC chapters and ATC codes were calculated. ICPC chapters that differed significantly between CRC patients and controls were further analysed. To get an impression whether differences were due to symptoms or diagnoses, the researchers assessed whether differences on these chapters were due to symptom (ICPC 1-29) or diagnostic codes (ICPC 70-99).

Statistical analysis. Due to the overdispersion in the healthcare data, a negative binomial regression model was fitted with a robust covariance matrix of the exchangeable type, thus accounting for clustering in the data due to the matching procedure. Log-transformed observation time was included in the model as an offset variable to account for differences in observation times. Annual incidence rate ratios (IRR) and 95\% confidence intervals (CI) were presented.

\section{Qualitative analysis}

Symptoms. Because GPs may have coded only one complaint of patients with CRC, two researchers extracted and descriptively counted all complaints in the abdominal/ pelvic region, and general complaints (for example, tiredness, fever, or feeling ill), from the free-text subjective data fields.

Qualitative coding. Coding of the complete SOAP texts was performed by two researchers. First, texts were conscientiously read and re-read by the researchers, potentially interesting text was marked, and descriptive codes were assigned to these segments by researchers 
Table 1. Characteristics of patients with colorectal cancer, reference population, and characteristics of the purposive sample for qualitative analysis

\begin{tabular}{|c|c|c|c|}
\hline Characteristic & $\begin{array}{l}\text { Patients } \\
\text { with CRC } \\
\text { (N=287) }\end{array}$ & $\begin{array}{c}\text { Reference } \\
\text { population } \\
\text { (N=828) }\end{array}$ & $\begin{array}{c}\text { Purposive } \\
\text { Sample } \\
\text { (N=57) }\end{array}$ \\
\hline \multicolumn{4}{|l|}{ Sex, $n(\%)$} \\
\hline Male & 146 (50.9) & $423(51.1)$ & 29 (50.8) \\
\hline Female & 141 (49.1) & 405 (48.9) & $28(49.1)$ \\
\hline Mean age at diagnosis, years (SD) & $68.8(12.2)$ & $68.3(12.0)$ & $68.1(12.5)$ \\
\hline \multicolumn{4}{|l|}{ Tumour location, $n(\%)$} \\
\hline Colon & 187 (65.2) & & $39(68.4)$ \\
\hline Rectum & 74 (25.8) & & $16(28.1)$ \\
\hline Unknown & $26(9.1)$ & & 2 (3.5) \\
\hline \multicolumn{4}{|l|}{ TNM stage at diagnosis, $n(\%)$} \\
\hline $0 / 1 / I 1$ & 97 (33.8) & & 19 (33.3) \\
\hline III/IV & $128(44.6)$ & & $27(47.4)$ \\
\hline Unknown & $62(21.6)$ & & 11 (19.3) \\
\hline Face-to-face contact, median (IQR) & & & $5.0(2.0-9.5)$ \\
\hline \multicolumn{4}{|l|}{ Practice, $n[\%]$} \\
\hline Groningen & & & $18(31.6)$ \\
\hline Sappemeer & & & 21 (36.8) \\
\hline Hoogeveen & & & $18(31.6)$ \\
\hline
\end{tabular}

independently, for example, inductively explored. ${ }^{20}$ These segments and codes were discussed between the two researchers until consensus was reached. In case the researchers did not agree, or were uncertain about contextual interpretation, a third researcher with a GP background was consulted. These codes were then interpreted, categorised, and clustered by all three researchers, thereby creating a thematic map. An iterative process was applied, meaning that the newly formed thematic map was applied to all data, until no new codes or themes emerged, and saturation was reached.

\section{Table 2. Comparison of the median number of face-to-face contacts and prescribed medication in the year prior to referral for colorectal cancer between patients with colorectal cancer, $N=287$, and reference population, $\mathbf{N}=\mathbf{8 2 8}$}

\begin{tabular}{lcc} 
Population & Median (IQR) & IRR (95\% Cl) \\
\hline Face-to-face contacts & $3.0(1.0-6.0)$ & 1.0 \\
Controls & $4.0(2.0-8.0)$ & $1.41(1.25$ to 1.59$)$ \\
Patients with CRC & & 1.0 \\
\hline Medication & $9.0(2.0-21.0)$ & $1.21(1.07$ to 1.37$)$ \\
$\quad$ Controls & $12.0(4.0-26.0)$ & \\
\hline
\end{tabular}

$C R C=$ colorectal cancer. $I Q R=$ interquartile range. $I R R=$ incidence rate ratio .

\section{RESULTS}

The authors identified 287 patients with a confirmed diagnosis of CRC (Figure 1). The characteristics of these patients $(n=287)$ and their matched controls ( $n=828)$ are shown in Table 1. Most patients with CRC $(n=187 ; 65.2 \%)$ were diagnosed with colon cancer, while 74 (25.8\%) were diagnosed with rectal cancer. Almost half were diagnosed with a tumour, node, and metastasis (TNM) stage III or IV (44.6\%), although staging was unknown for 62 (21.6\%) patients.

\section{Quantitative analysis of healthcare use}

In the year before the diagnosis, patients with CRC had a significantly higher rate of GP contact (IRR $1.41,95 \% \mathrm{Cl}=1.25$ to 1.59) compared with controls; a median (IQR) of $4.0(2.0-8.0)$ versus $3.0(1.0-6.0)$, respectively (Table 2). In this period, patients with CRC were also prescribed significantly more medication compared with controls (IRR $1.21,95 \% \mathrm{Cl}=1.07$ to 1.37 ).

Patients with CRC showed significantly more contacts coded as general and unspecified reasons (ICPC-A, IRR 1.39, $95 \% \mathrm{Cl}=1.04$ to 1.86 ); digestive (ICPC-D, IRR 3.93, $95 \% \quad \mathrm{Cl}=3.06$ to 5.05); musculoskeletal (ICPC-L, IRR 1.42, $95 \% \mathrm{Cl}=1.10$ to 1.84); neurological (ICPC-N, IRR $1.70,95 \% \mathrm{Cl}=1.03$ to 2.81 ); respiratory (ICPC-R, IRR $1.46,95 \% \mathrm{Cl}=1.08$ to 1.97 ); and endocrine, metabolic, and nutritional reasons (ICPC-T, IRR 1.56, 95\% Cl = 1.09 to 2.23) in the year before diagnosis, compared with controls (Table 3). The largest difference was observed for the digestive system: $46.0 \%$ of patients with CRC showed two or more contacts for these reasons in the year before diagnosis, compared with $12.2 \%$ of controls (data not shown). The most prevalent ICPC-D codes were: unspecified abdominal pain (D06, 13.7\% of all ICPCD codes), constipation (D12, 13.0\%), and stomach pain (D02, 7.6\%). Further analysis (Figure 2) of the ICPC chapters that differed significantly between patients with CRC and their controls revealed that, for ICPC chapters A (general/unspecified), $\mathrm{D}$ (digestive system), L (musculoskeletal), and $\mathrm{N}$ (neurological), more symptom codes were found (ICPC 1-30). For ICPC chapters $\mathrm{R}$ (respiratory) and $\mathrm{T}$ (endocrine, metabolic, and nutritional), more diagnoses were coded (ICPC 70-99; Figure 2).

Table 3 shows differences in prescribed medication between patients with CRC and controls. Significantly more medication was prescribed in the year before diagnosis for the alimentary tract and metabolism 


\section{Table 3. Reasons for primary healthcare use among patients with colorectal cancer $(N=287)$ and patients from the reference population $(N=828)$ in the year before referral}

\begin{tabular}{|c|c|c|c|}
\hline Reason & $\begin{array}{c}\text { Reference } \\
\text { population, rate } \\
\text { (N=828) }\end{array}$ & $\begin{array}{c}\text { CRC patients, } \\
\text { rate }^{a} \\
(N=287)\end{array}$ & $\begin{array}{c}\text { IRR } \\
(95 \% \mathrm{CI})^{\mathrm{b}}\end{array}$ \\
\hline \multicolumn{4}{|l|}{ Face-to-face consults (ICPC chapter) } \\
\hline General and unspecified (A) & 0.45 & 0.60 & 1.39 (1.04 to 1.86$)$ \\
\hline Digestive (D) & 0.59 & 2.22 & 3.93 (3.06 to 5.05 ) \\
\hline Musculoskeletal (L) & 1.24 & 1.73 & $1.42(1.10$ to 1.84$)$ \\
\hline Neurological (N) & 0.20 & 0.33 & 1.70 (1.03 to 2.81$)$ \\
\hline Endocrine, metabolic, and nutritional (T) & 0.61 & 0.92 & 1.56 (1.09 to 2.23 ) \\
\hline \multicolumn{4}{|c|}{ Prescribed medication (ATC Classification System chapter) } \\
\hline Alimentary tract and metabolism (A) & 1.04 & 2.87 & $1.25(1.02$ to 1.54$)$ \\
\hline Cardiovascular system (C) & 4.33 & 5.45 & 1.24 (1.07 to 1.43$)$ \\
\hline Anti-infectives for systemic use $(\mathrm{J})$ & 0.99 & 1.25 & 1.25 (1.03 to 1.51$)$ \\
\hline Musculoskeletal (M) & 0.70 & 1.03 & 1.45 (1.05 to 1.99$)$ \\
\hline Respiratory system (R) & 1.10 & 1.64 & 1.46 (1.01 to 2.13 ) \\
\hline
\end{tabular}

Figure 2. Distribution of proportion of ICPC codes within ICPC chapters for patients with CRC and controls in the year before referral. ICPC codes ranging 1-29 are symptoms whereas codes ranging 70-99 are diagnoses.

$C R C=$ colorectal cancer. $I C P C=$ International Classification of Primary Care.
(ATC-A, IRR $1.25,95 \% \mathrm{Cl}=1.02$ to 1.54 ), the cardiovascular system (ATC-C, IRR $1.24,95 \% \mathrm{Cl}=1.07$ to 1.43$)$, anti-infectives for systemic use (ATC-J, IRR 1.25 $95 \% \mathrm{Cl}=1.03$ to 1.51), the musculoskeletal system (ATC-M, IRR: 1.45, 95\% Cl = 1.05 to 1.99), and respiratory system (ATC-R, IRR $1.46,95 \% \mathrm{Cl}=1.01$ to 2.13 ).

\section{Qualitative content analysis of SOAP text}

Table 1 shows the characteristics of the purposive sample of 57 patients with CRC and the location of the practice of the 25 GPs who were included in the qualitative content analysis of the SOAP texts. Saturation was reached after analysis of 48 electronic

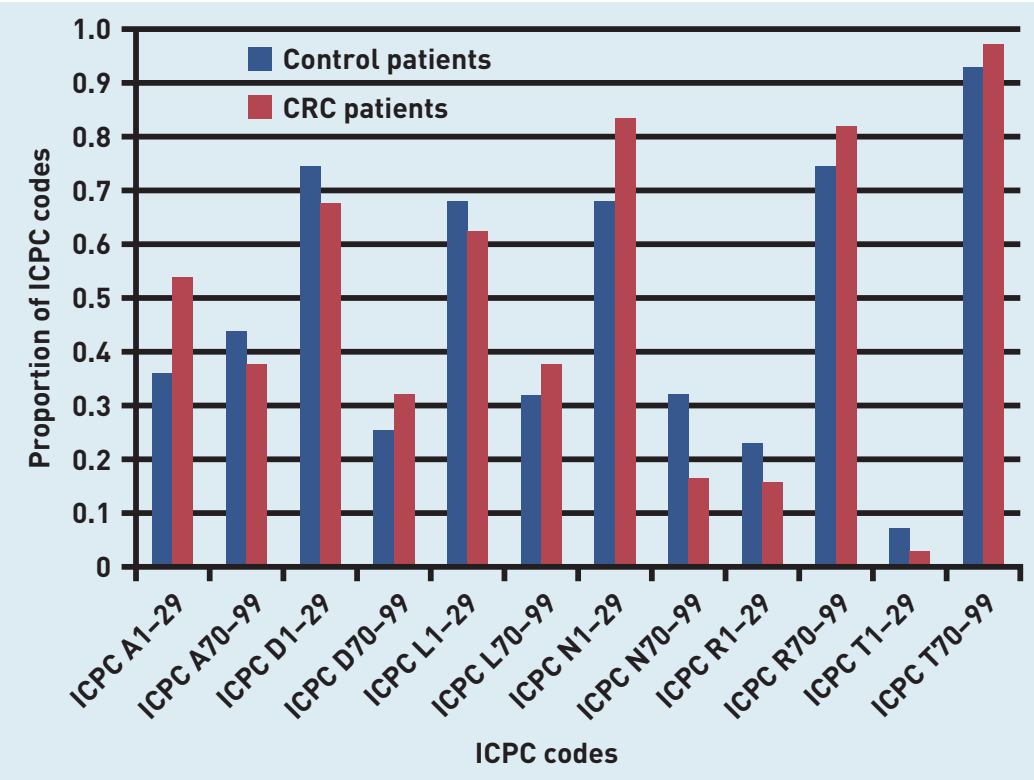

patient records; no new information was obtained from nine subsequent medical records.

Symptoms. From the subjective (S) data of the 57 patients with CRC during the year before referral 210 presenting symptoms were coded (Figure 3). The most prevalent symptoms were abdominal pain (number of complaints $=28$ ), blood/slime in faeces ( $n=21)$, diarrhoea $(n=19)$, and change in bowel habits $(n=19)$. Less specific symptoms such as stomach complaints/ vomiting ( $n=13)$, back pain ( $n=12)$, fatigue ( $n=10)$, flatulence, bloating, or rumbling in the abdominal area $(n=8)$ were less frequently recorded.

Two major themes emerged during the qualitative analysis. The first comprised cases where missed diagnostic opportunities' may have occurred, leading to less timely diagnoses $(n=35)$. The second comprised cases where no improvement in the diagnostic process could have been expected $(n=22)$

Theme 1: possible missed diagnostic opportunities. Possible missed diagnostic opportunities, as derived from the qualitative analysis of electronic patient records, may either be attributed to patient factors or doctor factors.

Patient factors included many patients appearing to wait for prolonged periods before presenting themselves with symptoms they experienced. Furthermore, patients who visited their GP frequently did not always mention complaints related to their bowels when they occurred, but only 


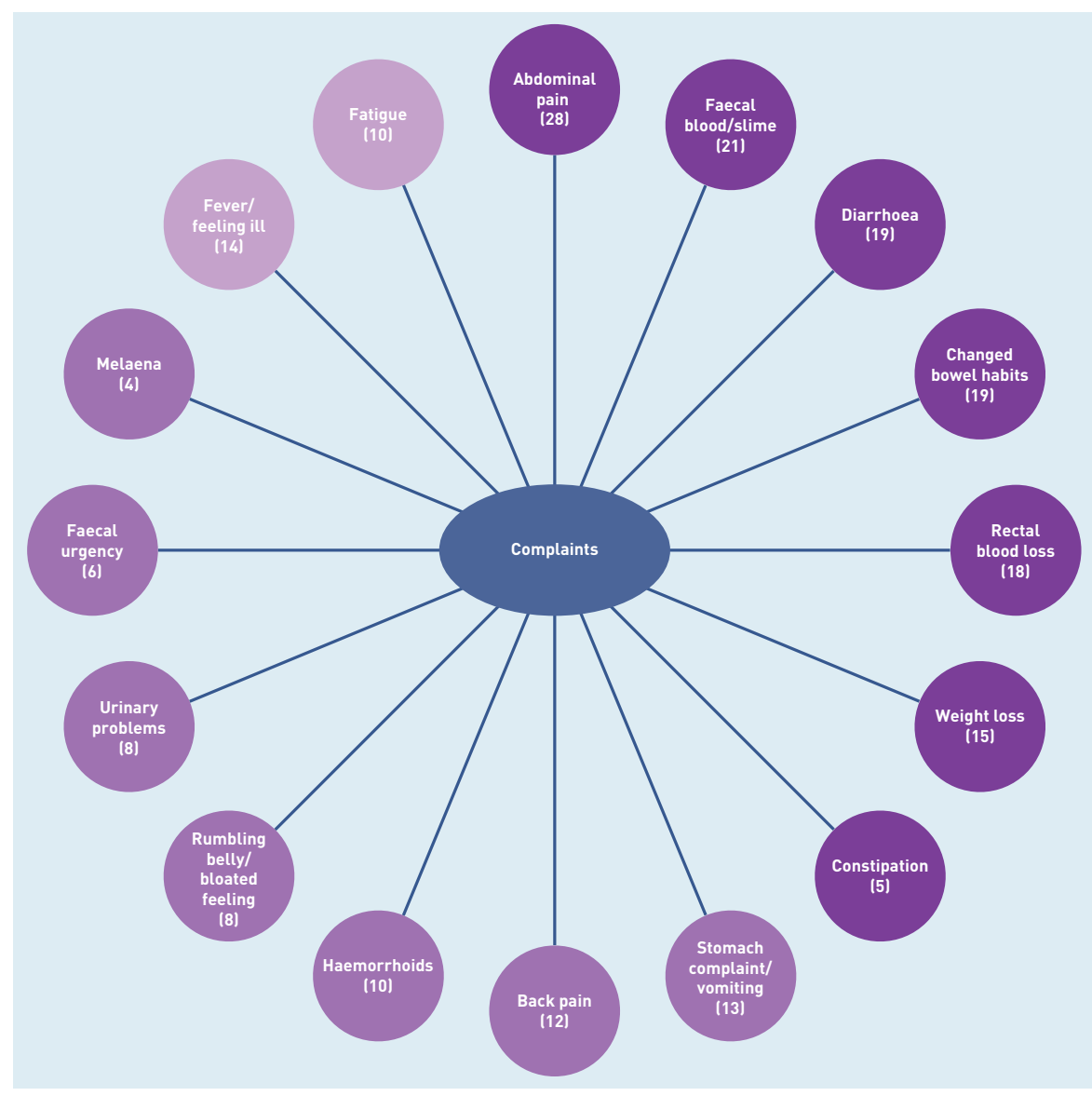

Figure 3. Complaints, mentioned in electronic patient records of the purposive sample, $n=57$, in the year before referral. Figures in brackets indicate number of complaints, total complaints $=210$. The dark purple complaints are the well known 'alarm symptoms'. The mid-purple complaints are less specific in the abdominal area. The pale purple complaints are general and not related to the abdominal area. after some time. For example, a patient who had visited the GP for various reasons, such as eczema, coughing, and diabetes, at one point mentioned they also had daefecation problems:

'For a prolonged time (+/- 1 year) increasing defaecation problems, alternating pattern, sometimes slimy and with blood.' IS data field, Male [M], 70 years)

Sometimes patients cancelled appointments when complaints had diminished, which led to longer intervals when complaints returned. One patient, who was scheduled for a colonoscopy, cancelled that appointment because their symptoms had gone. Longer intervals were also due to circumstances in patients' lives. Patients' worries about their home situation, problems experienced by their partner, or other situations in their lives, also led to a later presentation of their own health problems. For example, a female patient who had been consulting for diarrhoea and other abdominal complaints appeared to stop doing that for a couple of months because a situation with her husband interfered:
'Panicked, cannot deal with illness of husband and missing care of children, hyperemotional.' (S data field, Female [F], 84 years)

Examples of doctor factors were where, in many cases, GPs appeared to attribute symptoms to pre-existing conditions. For example, the symptoms of a patient who presented with lower abdominal pain and a history of diverticulitis were, at first, attributed to that condition. Another patient with persistent abdominal pain was referred to their gynaecologist because of a gynaecological history. Other abdominal or pelvic illnesses also seemed to mask the symptoms, for example, a patient who tested positive for a urinary tract infection (UTI) had a possible missed diagnostic opportunity after receiving test results and subsequent UTI treatment. The UTI diagnosis possibly did not explain all of the symptoms.

The intermittent nature of symptoms also led to longer diagnostic intervals in primary care. Non-persistent pain, and episodes of diarrhoea, or obstipation, all made it difficult to pinpoint the exact nature of these complaints. Some GPs did not immediately think of a CRC diagnosis in cases where patients presented with vague complaints, such as ongoing tiredness or prolonged and unexplained fever.

GPs also seemed susceptible to their patients' explanations for the reasons behind the symptoms. Patients reported symptoms that could be attributed to CRC, but they gave good explanations of other causes. Their GP tended to accept these explanations and did not investigate the symptoms further. For example, a patient reported melaena and thought it was caused by their medication.

In some cases, GPs seemed to attribute possible symptoms of CRC to side effects of medication. For one patient, who consistently complained for 2.5 months over three visits about having 'moist flatulence' and was referred/diagnosed 5 months after onset, the GP coded

Still problems with metoprolol lagain moist flatulence), did not take medication, last 10 days little complaints -> let's try enalapril. ( $P$ data field, $M, 64$ years, rectal cancer)

Consultation behaviour by patients may also have contributed to prolonged diagnostic intervals. Patients who presented themselves frequently to their GP, with a variety of complaints, seemed to be less frequently referred for further investigation. Specialists also appeared to 
miss diagnostic opportunities. For example, a gastroenterologist coded:

Black defaecation with red colouring. Probably gastrointestinal bleeding, check $\mathrm{Hb}$ again in 2 months?' 10 data field, $\mathrm{M}$, 74 years, colon cancer)

In some cases, GPs stuck to their own preliminary diagnosis and did not re-evaluate their patient during subsequent consultations. This was also the case when one of the colleagues, in a case where there were multiple GP workers, had made a preliminary diagnosis, and another GP would carry on that line of thinking and not re-evaluate it.

Theme 2: improvements in diagnostic process unlikely. Among the 22 patients with CRC in this group, four did not have a GP involved in the diagnostic process. Of these, two were diagnosed by the national screening programme. Other patients presented themselves at emergency hospital care or were referred within hospital care. The remainder mainly had swift referrals, so these notes were markedly shorter.

It frequently appeared that the GP had correctly interpreted symptoms and quickly referred to secondary care for diagnosis. In many cases patients were quickly referred because of the fact that all 'red flag symptoms' had presented. These seemed to be patients who had not visited their GP in the last year, and presented with more than one alarm symptom. For example, a GP coded:

'Lost a lot of weight (6 kg) in short period, heavy faecal blood loss. Since 6 weeks tumultuous bowel, no pain. Frequent urgencies. Haemorrhoids around rectum. No deviances with rectal toucher. Decided to refer.' (M, 56 years, rectal cancer)

\section{DISCUSSION}

\section{Summary}

In the year before referral patients diagnosed with CRC had $41 \%$ more faceto-face contacts and $21 \%$ more medication prescriptions in general practice compared with an age-, sex-, and GP-matched reference population. Before diagnosis, patients with CRC showed significantly more contacts for general reasons, including symptoms relating to the digestive, musculoskeletal, neurological, respiratory, endocrine, metabolic, and nutritional systems; the largest difference was observed for the digestive system. Of the patients with CRC, $46 \%(n=132)$ had two or more contacts for these reasons, compared with $12.2 \%(n=101)$ of controls. Patients diagnosed with CRC were prescribed more medication in the year before their referral for the alimentary tract, but also more cardiovascular, musculoskeletal, and respiratory medication. Furthermore, patients with CRC presented not only with the known alarm symptoms but also with a variety of other, less clear symptoms such as back pain, rumbling in the abdominal area, flatulence, or general ill feeling.

The authors' qualitative analysis suggested that patient factors, such as prolonged periods before mentioning symptoms, or because they had been put at ease after symptoms diminished, could lead to missed diagnostic opportunities. Similarly, doctor factors, such as GPs attributing symptoms to comorbid conditions, medication use, the explanation from their patient about their symptoms, or because they stuck to an initial diagnosis, could also contribute to missed diagnostic opportunities. In other cases, improvements in the diagnostic process within general practice were not possible, mainly because the GP acted swiftly on alarm symptoms.

\section{Strengths and limitations}

A strength of this study is the multimethods approach. The quantitative findings in this study were enriched by using both quantitative and qualitative data to obtain a deeper understanding of the diagnostic process of detecting CRC in primary care. A prospective primary care database was also used where the presentation of healthcare problems and prescriptions were recorded by GPs using a standardised registration protocol, reducing the risk of recall or non-response bias compared with patient-reported data. ${ }^{21}$ The likelihood that the data concerning CRC diagnosis was correctly recorded by GPs was increased by revalidating the data with specialist letters in GP practices and hospital records. Moreover, this provided detailed information about type of treatment, tumour location, and tumour stage. Because not all correspondence from the reference population was studied, it cannot be ruled out that some controls had a history of CRC that was not coded in the RNG database. However, this would underestimate the differences found in healthcare use in this current study.

Although the quantitative data in this study were enriched with a qualitative analysis of the free texts of the electronic patient records, it has to be noted that 
these records are made by the GP They are, therefore, likely to be the GP's interpretation of symptoms mentioned by the patient, rather than a full representation of the patient perspective or what actually happened in the consultation room.

\section{Comparison with existing literature}

In line with this study, earlier research showed an increased consultation rate in general practice for patients with CRC before their diagnosis. ${ }^{14,16,22}$ Many patients with CRC in this study (46\%) had two or more contacts for digestive reasons, compared with only $12 \%$ of controls. This is in accordance with known alarm symptoms, such as rectal blood loss, constipation, diarrhoea, abdominal pain or tenderness, and weight loss. ${ }^{9-11}$ As these alarm symptoms as single symptoms do not have a high positive predictive power, ${ }^{11}$ repeated digestive complaints may warrant further investigation.

Interestingly, patients also had more contacts for respiratory, endocrine, metabolic, and nutritional reasons. The codes in these ICPC chapters were mainly diagnoses. It is known that comorbid conditions such as chronic obstructive pulmonary disease and diabetes are common among patients with CRC. ${ }^{23}$ Moreover, these conditions share lifestylerelated risk factors with CRC. ${ }^{24}$ This may also explain the study findings of higher rates of respiratory, musculoskeletal, and cardiovascular drugs among patients with CRC.

Moreover, patients do not exclusively present with these alarm symptoms, which are often the result of a coded interpretation by doctors and researchers of predefined symptoms. Whereas, according to this qualitative analysis, patients seemed to present with a variety of vague complaints in the abdominal and pelvic region, and other general complaints symptoms. Vague complaints may explain that patients had more contacts for general and unspecified reasons and the musculoskeletal system.

In an effort to create a standardised set of definitions related to key time points and intervals in the diagnosis of cancer the Aarhus Statement was developed. ${ }^{25}$ This distinguished the patient interval and doctor, or diagnostic, interval during the diagnostic process. Within these intervals, missed diagnostic opportunities can occur, which could cause delays in the diagnostic process. ${ }^{26}$ In this study there appeared to be such missed diagnostic opportunities when patients appeared not to mention abdominal problems, for example, some patients report later on that they have had abdominal problems for a period of time but fail to mention it earlier. Earlier research suggests that patients try to attribute their symptoms to benign conditions to retain normality, or do not mention them because of the private nature of the complaints. ${ }^{27}$ The researchers also observed that patients cancelled appointments in secondary care, for example, for colonoscopy, when complaints they had diminished; the invasive and private nature of the investigations might lead to this patient decision. In order to improve potential missed diagnostic opportunities, GPs could monitor whether patients attend appointments and investigations after referral.

GPs often appeared to attribute symptoms to other known illnesses, comorbid conditions, or medication use of their patients; this could interfere with a swift referral trajectory. GPs ordered tests (for example, for urinary infections), changed medication (for example, in relation to melaenal, or referred patients to other specialists based on their medical history. This could lead to a 'restart' of the diagnostic process, creating the opportunity for new patient factors: patients have to evaluate whether they are feeling better or at what point they need to consult their GP again. Combined with the intermittent nature of some complaints like diarrhoea, this may prolong diagnostic intervals profoundly. In these cases, good safety-netting strategies ${ }^{28}$ could reduce these delays. If patients are instructed about the uncertainty of preliminary diagnoses and are given information about well-defined clinical signs they have to look out for, re-consultation and subsequent diagnosis might be expedited.

Some GPs also did not appear to reconsider their initial diagnosis during subsequent consultations. They also sometimes seemed to unquestioningly accept explanations and/or diagnoses from colleagues and even from patients.

\section{Implications for practice}

Increased consultation rates for digestive problems, not necessarily alarm symptoms, may warrant further investigation into possible CRC. Although in most cases the diagnostic trajectory in primary care may not be expedited, there are pitfalls that GPs need to be aware of. These include the assumption that symptoms are caused by comorbid conditions or medication, or relating complaints to pre-existing medical conditions. GPs also need to be aware that repeated complaints warrant rethinking earlier diagnoses. 


\section{REFERENCES}

1. Torre LA, Bray F, Siegel RL, et al. Global cancer statistics, 2012. CA Cancer J Clin 2015; 65(2): 87-108.

2. Weller D, Coleman D, Robertson R, et al. The UK colorectal cancer screening pilot: results of the second round of screening in England. Br J Cancer 2007; 97(12): 1601-1605.

3. Hamilton W. Five misconceptions in cancer diagnosis. Br J Gen Pract 2009; DOI: https://doi.org/10.3399/bjgp09X420860.

4. Moss SM, Campbell C, Melia J, et al. Performance measures in three rounds of the English bowel cancer screening pilot. Gut 2012; 61(1): 101-107.

5. Gatta G, Capocaccia R, Sant M, et al. Understanding variations in survival for colorectal cancer in Europe: a EUROCARE high resolution study. Gut 2000; 47(4): 533-538.

6. Richards MA. The size of the prize for earlier diagnosis of cancer in England. Br J Cancer 2009; 101(Suppl 2): S125-S129.

7. Torring ML, Frydenberg M, Hamilton W, et al. Diagnostic interval and mortality in colorectal cancer: $\mathrm{U}$-shaped association demonstrated for three different datasets. J Clin Epidemiol 2012; 65(6): 669-678.

8. Sikdar KC, Dickinson J, Winget M. Factors associated with mode of colorectal cancer detection and time to diagnosis: a population level study. BMC Health Serv Res 2017; 17(1): 7.

9. Hamilton W, Round A, Sharp D, Peters TJ. Clinical features of colorectal cancer before diagnosis: a population-based case-control study. Br J Cancer 2005; 93(4): 399-405.

10. Hamilton W. The CAPER studies: five case-control studies aimed at identifying and quantifying the risk of cancer in symptomatic primary care patients. $\mathrm{Br} J$ Cancer 2009; 101(Suppl 2): S80-S86.

11. Astin M, Griffin T, Neal RD, et al. The diagnostic value of symptoms for colorectal cancer in primary care: a systematic review. Br J Gen Pract 2011; DOI: https://doi.org/10.3399/bjgp11X572427.

12. Walter FM, Emery JD, Mendonca S, et al. Symptoms and patient factors associated with longer time to diagnosis for colorectal cancer: results from a prospective cohort study. Br J Cancer 2016; 115(5): 533-541.

13. Holtedahl $K$, Vedsted $P$, Borgquist $L$, et al. Abdominal symptoms in general practice: frequency, cancer suspicions raised, and actions taken by GPs in six European countries. Cohort study with prospective registration of cancer. Heliyon 2017; 3(6): e00328.

14. Christensen KG, Fenger-Grøn M, Flarup KR, Vedsted P. Use of general practice, diagnostic investigations and hospital services before and after cancer diagnosis - a population-based nationwide registry study of 127,000 incident adult cancer patients. BMC Health Serv Res 2012 ; 12: 224.

15. Ewing M, Naredi $P$, Nemes $S$, Zhang $C$, et al. Increased consultation frequency in primary care, a risk marker for cancer: a case-control study. Scand J Prim Health Care 2016; 34(2): 205-212.

16. Hansen PL, Hjertholm P, Vedsted P. Increased diagnostic activity in general practice during the year preceding colorectal cancer diagnosis. Int J Cancer 2015; 137(3): 615-624

17. van Wayenburg CA, van de Laar FA, de Waal MW, et al. Nutritional deficiency in Dutch primary care: data from general practice research and registration networks. Eur J Clin Nutr 2005; 59(Suppl 1): S187-S194.

18. Lamberts $\mathrm{H}$, Wood M, eds. ICPC. International classification of primary care. 1st edn. Oxford: Oxford University Press,1987.

19. WHO Collaborating Centre for Drug Statistics Methodology. ATC index with DDDs. Oslo: WHO CCDSM, 1999.

20. Pope C, Ziebland S, Mays N. Qualitative research in health care. Analysing qualitative data. BMJ 2000; 320(7227): 114-116.

21. Tisnado DM, Adams JL, Liu H, et al. What is the concordance between the medical record and patient self-report as data sources for ambulatory care? Med Care 2006; 44(2): 132-140.

22. Brandenbarg D, Roorda C, Groenhof F, et al. Increased primary health care use in the first year after colorectal cancer diagnosis. Scand J Prim Health Care 2014; 32(2): 55-61.

23. van Leersum NJ, Janssen-Heijnen ML, Wouters MW, et al. Increasing prevalence of comorbidity in patients with colorectal cancer in the South of the Netherlands 1995-2010. Int J Cancer 2013; 132(9): 2157-2163.

24. Lin OS. Acquired risk factors for colorectal cancer. Methods Mol Biol 2009; 472: $361-372$

25. Weller D, Vedsted P, Rubin G, et al. The Aarhus statement: improving design and reporting of studies on early cancer diagnosis. Br J Cancer 2012; 106(7): 1262-1267.

26. Lyratzopoulos G, Vedsted P, Singh H. Understanding missed opportunities for more timely diagnosis of cancer in symptomatic patients after presentation. Br J Cancer 2015; 12(Suppl 1): S84-S91.

27. Hall N, Birt L, Banks J, et al. Symptom appraisal and healthcare-seeking for symptoms suggestive of colorectal cancer: a qualitative study. BMJ Open 2015; 5(10): e008448.

28. Almond S, Mant D, Thompson M. Diagnostic safety-netting. Br J Gen Pract 2009; DOI: https://doi.org/10.3399/bjgp09X472971. 